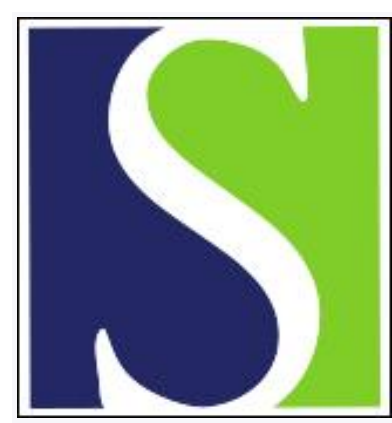

Scand J Work Environ Health 1993;19(4):291-293

https://doi.org/10.5271/sjweh.1471

Issue date: 01 Aug 1993

Meeting of the IARC working group on occupational exposures of hairdressers and barbers and on some colorings and dyestuffs.

by Vainio $\mathrm{H}$, McGregor DB, Heseltine $\mathrm{E}$

Affiliation: International Agency for Research on Cancer, Lyon, France.

This article in PubMed: www.ncbi.nlm.nih.gov/pubmed/8235519

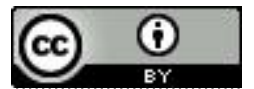




\section{Meeting of the IARC working group on occupational exposures of hairdressers and barbers and on some colorings and dyestuffs}

The 57th meeting of experts convened by the International Agency for Research on Cancer (IARC), within the program of the IARC Monographs on the Evaluation of Carcinogenic Risks to Humans, evaluated the evidence with regard to the risks of exposures of hairdressers, barbers, and users of hair coloring materials. The Working Group (list of participants at the end of this article) also looked at literature on eight nitro aromatic amines used in hair coloring formulations and literature on agents related to the main theme of the meeting - one cosmetic coloring agent, four industrial dyestuffs, and four aromatic amines, including 4,4'-methylene bis(2-chloroaniline) (MOCA).

\section{Exposures of hairdressers, barbers and personal users of hair coloring materials}

The occupational exposures of hairdressers and barbers are difficult to define. People working in these professions can be exposed to over 5000 chemical products in their work, both during hair treatments such as shampooing, styling, waving, straightening, bleaching, and dyeing and during the use of skin and nail products. The terms hairdresser, beautician, and cosmetologist are often used interchangeably. However, although hairdressers in the United States are involved primarily in the care of women's hair, in the United Kingdom, for instance, the term can also include barbers. The term barber has usually been applied to people who cut men's hair. Barbers can also apply hair sprays and other styling preparations and shave facial hair. Furthermore, in some countries barbers are almost exclusively men, whereas in other countries a large proportion comprises women.

The actual exposures of home users of hair colorants are also unclear, as home users employ a wide spectrum of colors and types of treatment made up of a variety of chemical products.

Hair colorants are classified as permanent (constituting primarily aromatic amines and aminophenols with hydrogen peroxide), semipermanent (nitrosubstituted aromatic amines, aminophenols, aminoanthraquinones and azo dyes), and temporary (highmolecular-weight or insoluble complexes and metal salts, such as lead acetate). Temporary colorants are not used to a significant extent by hairdressers.

The compositions of many of the preparations used by both professional and private users of hair treatments have changed with time so that studies of exposures 20 or 30 years ago may not be relevant to current exposures. Problems of this nature are encountered in most studies of occupational cancers.

The use of hair coloring materials is widespread. About $35 \%$ of women and $10 \%$ of men in Europe, Japan, and the United States use hair colorants. The Working Group convened by IARC thus considered all of the epidemiologic studies addressing the professions of hairdresser, barber, beautician, and cosmetologist and also those that looked at the private use of hair coloring materials. It found consistent evidence from five out of six large cohort studies, all conducted in Europe, of an excess risk of cancer of the urinary bladder among male hairdressers and barbers. The increase was significant in three of the studies, and the overall risk relative to that in the general population was about 1.6. In 12 case-referent studies, male hairdressers and barbers had an overall relative risk for urinary bladder cancer of about 1.2. The possibility that the excess was due to a higher prevalence of tobacco smokers in this professional group was considered. In the three case-referent studies in which smoking was adjusted for, no excess risk for urinary bladder cancer was found. The cohort studies, however, showed an overall relative risk of about 1.3 for lung cancer among male and female hairdressers. Thus the higher prevalence of smokers reported among male hairdressers and barbers in some studies is consistent with the overall excess of lung cancer, but it cannot readily explain the magnitude of the increase in risk for cancer of the urinary bladder in the cohort studies. Furthermore, the studies in Denmark and Switzerland showed significant excesses of urinary bladder cancer unaccompanied by appreciable excesses of lung cancer.

The risk for cancer of the urinary bladder was less consistently increased in corresponding studies of women. Negative results were found in three studies, and positive results were obtained in five cohort studies. None of the results in the studies of women were statistically significant.

The only other cancer type that appeared in excess among hairdressers and barbers was non-Hodgkin's lymphoma. One case-referent study from Australia found a significant excess risk for non-Hodgkin's lymphoma, this time among female hairdressers. Nonsignificant excesses of this malignancy were noted among men and women in one cohort study from Denmark and among men in one casereferent study from the United States.

The association between personal use of hair coloring materials and urinary bladder cancer has 
been studied in seven case-referent studies. Overall, these investigations do not indicate an excess risk. After the appearance of a report in 1976 that women who used hair dyes had an excess risk of breast cancer, one cohort and five case-referent studies were conducted; there was no excess overall. The suggestion of an excess risk for non-Hodgkin's lymphoma among professional hairdressers was reflected, however, in the finding of a significant excess risk for this tumor type among male users of hair coloring products in one case-referent study and among female users in another.

In arriving at an overall evaluation of carcinogenicity, IARC working groups not only consider epidemiologic evidence, but also evaluate that from experimental systems. The available studies of commercially available hair dyes or laboratory mixtures of hair dyes on experimental animals were, however, considered to be inappropriate for evaluating the systemic carcinogenicity of the formulations, as the experiments were designed to evaluate skin toxicity and the doses used were nontoxic. On the basis of the limited evidence in the epidemiologic studies of an excess risk of bladder cancer among male hairdressers and barbers, the Working Group therefore concluded that occupation as a hairdresser or barber entails exposures that are probably carcinogenic (group $2 \mathrm{~A}$ in the IARC classification), whereas personal use of hair coloring products is not classifiable as to its carcinogenicity (group 3).

\section{Hair dyes and cosmetic colors}

In an attempt to establish whether the specific hair dyes to which hairdressers and, less often, barbers are exposed are responsible for any excess cancer risk, the literature was searched for studies in which the carcinogenicity of such compounds had been examined. The following eight compounds were selected for review and evaluation: CI Acid Orange 3, HC Blue No 1, HC Blue No 2, HC Red No 3, HC Yellow No 4, 2-amino-4-nitrophenol, 2-amino-5-nitrophenol, and 1,4-diamino-2-nitrobenzene. The available evidence did not allow seven of the eight dyes to be classified as to their carcinogenicity to humans (group 3), and only $\mathrm{HC}$ Blue No 1, the production and use of which were discontinued in the mid1980 s, was considered to be possibly carcinogenic to humans (group 2B). Neither could D \& C Red No 9 , used as a coloring agent in lipsticks, mouthwashes, dentifrices, and drugs, be classified as to its carcinogenicity to humans on the basis of the available experimental information, although it induced splenic sarcomas in male rats in one study.

\section{Industrial dyestuffs}

The Working Group also evaluated evidence for the carcinogenicity of four industrial dyestuffs. Magenta, a variable mixture prepared from aniline and ortho-toluidine, was first produced commercially in
Germany in the late 19th century. It has been used in textile dyes, printing inks, and biological stains. Marked excess risks for cancer of the urinary bladder have been observed in two small cohorts of workers in magenta production. This finding led the Working Group to conclude, as had a previous working group of IARC, that the manufacture of magenta entails exposures that are carcinogenic (group 1). The concomitant exposures of the workers to other aromatic amines in their work environment made it difficult to evaluate the carcinogenicity of magenta itself. However, since a known component of magenta, CI Basic Red 9, is clearly carcinogenic to experimental animals, the Working Group additionally concluded that magenta containing this compound is possibly carcinogenic to humans (group $2 \mathrm{~B}$ ).

CI Direct Blue 15, CI Acid Red 114 and CI Pigment Red 3 are widely used industrial azo dyes. The first two, which are derived from benzidine congeners, were considered on the basis of sufficient experimental evidence of carcinogenicity to be possibly carcinogenic to humans (group 2B). CI Pigment Red 3 could not be classified as to its carcinogenicity to humans (group 3 ).

\section{Aromatic amines}

The Working Group finally considered four aromatic amines. para-Chloroaniline, 2,6-dimethylaniline, and $\mathrm{N}, \mathrm{N}$-dimethylaniline are used in the manufacture of dyestuffs and have other applications, such as in the manufacture of agrochemicals and pharmaceuticals. No epidemiologic study was available that addressed exposures to these three compounds. On the basis of sufficient evidence of carcinogenicity in experimental animals, para-chloroaniline and 2,6-dimethylaniline were evaluated as possibly carcinogenic to humans (group 2B). $N, N$-dimethylaniline could not be classified as to its carcinogenicity (group 3).

The fourth aromatic amine considered was 4,4'methylene bis(2-chloroaniline) (MOCA), used principally as a curing agent in certain castable polyurethane products. It is used in many countries, is produced in quantities of several thousand tons a year, and has been released into the environment. The epidemiologic reports of exposures to MOCA can be classified only as descriptive studies. The results of analytical studies have not been published, although, in 1983, it was stated in a review that a cohort study was under way in a plant manufacturing MOCA in the United Kingdom. The first study of a cohort of workers who had potential contact with MOCA was reported in 1971, but urinary bladder cancer was not mentioned in the company records. The only other published study, carried out in the United States more recently, describeld the results of a screening procedure in which three asymptomatic tumors of the urinary bladder were identified, two of them in young workers. Unfortunately, the expected number of tumors at this site could not be calculated, as valid 
comparison rates of asymptomatic urinary bladder tumors were not available.

The results of experiments on the carcinogenicity of MOCA in animals show clearly that it is carcinogenic. Furthermore, MOCA forms adducts with deoxyribonucleic acid (DNA) both in vitro and in vivo. One of the two major adducts was not only found in the target tissues for carcinogenicity in exposed rats, but it was also co-chromatographed with a DNA adduct from urothelial cells recovered from the urine of a worker who was accidentally sprayed over his torso and upper extremities with several liters of molten MOCA in the polyurethane industry. MOCA is comprehensively genotoxic, and rats, dogs, and humans metabolize the compound in the same way. Although the available epidemiologic evidence is inadequate, this accumulation of evidence on the mechanisms of the carcinogenic action of MOCA in animals and the strong similarities of its properties in experimental systems and in human studies led the Working Group to conclude that MOCA is probably carcinogenic to humans (group 2A).

Dyes have been the object of epidemiologic scrutiny for almost a century, and studies of urinary bladder cancer among workers in the dyestuffs industry hold an important place in the history of occupationally related cancers. At least in most industrialized countries, the professions and industries in which exposures to dyes and aromatic amines occur have therefore been the object of stringent control measures so that exposures to these compounds have been minimized. The importance of this latest series of evaluations from IARC is that they can increase awareness of the potential hazards in other countries where these compounds are produced and used and lead these countries to take the necessary measures to reduce exposures.

\section{List of participants}

M Boeniger, National Institute for Occupational Safety and Health, Robert A Taft Laboratories, 4676 Columbia Parkway, Cincinnati, OH 45226-1998, USA

R Combes, LSU College of Higher Education, The Avenue, Southampton, Hants SO9 5HB, United Kingdom

TA Dragani, Division of Experimental Oncology A, Istituto Nazionale per lo Studio e la Cura dei Tumori, via Venezian 1, 20133 Milano, Italy

J Fitzgerald, Public and Environmental Health Division, South Australian Health Commission, 11-13 Hindmarsh Square, Adelaide, SA 5000, Australia

BD Hardin, National Institute for Occupational Safety and Health, Hubert H Humphrey Building, Room 714-B, 200 Independence Avenue SW, Washington, DC 20201, USA

RB Hayes, Division of Cancer Etiology, National Cancer Institute, Executive Plaza North, Room 418, 6130 Executive Boulevard, North Bethesda, MD 20892, USA

F Kadlubar, National Center for Toxicological Research,
Jefferson, AR 72079-9502, USA

LJ Kinlen, CRC Cancer Epidemiology Research Group, University of Oxford, The Radcliffe Infirmary, Oxford OX2 6HE, United Kingdom

E Kriek, Division of Molecular Carcinogenesis, The Netherlands Cancer Institute, Plesmanlaan 121, 1066 CX Amsterdam, The Netherlands

E Lynge, Danish Cancer Registry, Rosenvængets Hovedvej 35, Box 839, 2100 Kobenhavn $\emptyset$, Denmark

RR Maronpot, National Institute of Environmental Health Sciences, PO Box 12233, Research Triangle Park, NC 27709, USA

H-G Neumann, Institute of Pharmacology and Toxicology, University of Würzburg, Versbacherstrasse 9, 8700 Würzburg, Germany (Vice-Chairman)

S Olin, International Life Sciences Institutes, Risk Science Institute, 1126 Sixteenth Street, NW, Washington, DC 20036, USA

A Pintér, Department of Morphology, National Institute of Hygiene, Gyáli ut 2-6, 1097 Budapest, Hungary

U Rannug, Wallenberg Laboratory, University of Stockholm, 10691 Stockholm, Sweden

B Terracini, Dipartimento di Scienze Biomediche e Oncologia Umana, University of Torino, via Santena 7, 10126 Torino, Italy (Chairman)

H Tsuda, Second Department of Pathology, Fujita-Gakuen Health University, School of Medicine, Toyoake, Aichi 470-11, Japan

VS Turusov, Cancer Research Centre, Russian Academy of Medical Sciences, Kashirskoye Shosse 24, 115478 Moscow, Russia

\section{Representatives}

National Cancer Institute

P Wirth, Laboratory of Experimental Carcinogenesis, Division of Cancer Etiology, National Cancer Institute, Building 37, Room 3C-10, Bethesda, MD 20892, USA

Ecological and Toxicological Association of Dyes and Organic Pigments Manufacturers

EA Clarke, Ecological and Toxicological Association of Dyes and Organic Pigments Manufacturers, Clarastrasse 4, PO Box, 4005 Basel, Switzerland

R Jung, Hoechst AG, Pharma Forschung Toxicologie, Gewerbetoxicologie/GS 282, Postfach 8003 20, 6230 Frankfurt 80, Germany

Cosmetic, Toiletries and Fragrance Association and the Comité de Liaison des Associations européennes de l'Industrie de la Parfumerie, des Produits cosmetiques et de Toilette

JF Corbett, Clairol, 2 Blachley Road, Stamford, CT 06922 , USA

Commission of the European Communities

M De Smedt, Industrial Hygiene and Safety Unit V/E/2, Health and Safety Directorate, Commission of the European Communities, Bâtiment Jean Monnet, 2920 Luxembourg, Grand Duchy of Luxembourg

Harri Vainio, MD, Douglas B McGregor, PhD, Elisabeth Heseltine, MSc

International Agency for Research on Cancer

150 cours Albert Thomas

F-69372 Lyon Cédex 08, France 